\title{
High Speed, High Resolution pnCCDs as Two Dimensional Imaging Spectrometers for X-rays and Electrons
}

\author{
H. Soltau ${ }^{2}$, R. Hartmann ${ }^{1}$, P. Holl ${ }^{1}$, S. Ihle ${ }^{1}$, H. Ryll ${ }^{1}$, M. Huth ${ }^{1}$, J. Schmidt ${ }^{1}$, R. Eckhardt ${ }^{2}$, M. Simson ${ }^{2}$, \\ J. Soltau ${ }^{2}$, Ch. Thamm ${ }^{2}$, L. Strüder ${ }^{1}$ \\ ${ }^{1}$ PNSensor GmbH, Römerstr. 28, D-80803 Munich, Germany \\ 2 PNDetector GmbH, Emil-Nolde-Str.10, D-81735 Munich, Germany
}

Since several years pnCCDs are well known as radiation detectors for spectroscopic imaging in many fields of science: X-Ray Fluorescence analysis (XRF), X-ray astronomy, X-ray Free Electron Laser science, synchrotrons and in the visible range as wave front sensors in adaptive optics systems. For direct electron detection in the focal plane of (S)TEMs they have delivered remarkable results in an energy range from $20 \mathrm{keV}$ up to $300 \mathrm{keV}$.

pnCCDs are radiation detectors on high resistivity $450 \mu \mathrm{m}$ thick fully sensitive silicon. They are backilluminated devices with an ultra-thin, homogeneous radiation entrance window, enabling the proper detection of X-rays up to $30 \mathrm{keV}$ with high quantum efficiency. As all pnCCDs are equipped with a fully column parallel readout, frame rates on more than 1.000 frames per second are achieved, keeping the read noise level at 3 electrons (rms). Some of the key performance figures are e.g. a quantum efficiency above $90 \%$ from $1 \mathrm{keV}$ up to $10 \mathrm{keV}$, single photon counting capability starting at $30 \mathrm{eV}$ only and at $1 \mathrm{keV}$ for electrons, extreme radiation hardness due to the avoidance of active MOS structures, operation at temperatures around $-20^{\circ} \mathrm{C}$ or warmer, energy resolution of less than $125 \mathrm{eV}$ (FWHM) at $6 \mathrm{keV}$ and 37 $\mathrm{eV}(\mathrm{FWHM})$ at $90 \mathrm{eV}$. These properties have enabled a variety of spectacular measurements.

In the past 12 months this list of outstanding properties has been expanded significantly: (a) The X-ray colour camera has been reconfigured, resulting in a compact, light-weight camera, (b) a high dynamic range mode has been established with a charge handling capacity of more than 2.5 million signal charges in a single pixel, (c) a controlled charge extraction mode was successfully demonstrated, allowing to take out surplus charges in a pixel, to avoid the spilling over of excess charges in case of extreme illumination of a pixel and finally (d) the subpixel spatial resolution was improved substantially giving a $264 \times 264$ pnCCD pixel array more than $3 \mathrm{k} \times 3 \mathrm{k}$ resolution points. The focus of this presentation is to highlight the new achievements (a) to (d) starting from the already extraordinary basic device and system properties.

(a) New Colour X-ray camera: The new CXC (see Fig. 1) was designed to fit in small and tight surroundings. The new camera can be operated in vacuum without any entrance filter or with a Be filter in normal environments. Capillary optics can be coupled to the entrance window. The total weight of the camera head is $3 \mathrm{~kg}$. The round shaped camera has a diameter of $14 \mathrm{~cm}$ and a depth of $4 \mathrm{~cm}$. Cooling is performed through Peltier elements, which can be coupled - if needed - to a cold sink connected to a chilling system.

(b) High dynamic range mode: Device simulations have shown that the amount of signal charges, generated in the pnCCD through radiation to be stored in a single pixel can be increased by shifting the charge storage and transfer area closer to the transfer registers and by making the electrical surrounding of the charge storage location as negative as possible. This supports the confinement of the signal charge in a well-defined volume and therefore increases the charge handling capacity of a pixel. The previously 
applied standard operating modes were able to handle about 300.000 electrons in a single pixel. The new settings allow confining and transferring more than 2.5 million electrons in the CCD in a linear detection mode. In order not to exceed the dynamic range of the subsequent electronics, the gain of the amplifying stages has to be reduced. This can be done on-line through the programmable CAMEX ASIC amplifier array.

(c) Controlled charge extraction: If the amount of signal charge overcomes the charge handling capacity limit the surplus charges can be taken out in a controlled way to avoid overflowing electrons to spoil the information content of the neighbouring pixels. As we do have a direct electrical access to every single pixel we can define a saturation level of the pixels from when on additional charges are taken out (anti-blooming) (see Fig. 2). We have tested this mechanism experimentally with a charge load of 2 billion electrons per pixel, with a $1 \mathrm{~ms}$ frame time. An example is shown in Fig 3.

(d) Subpixel resolution: The very low noise of the pnCCD system at even high speed enables the user to centroid the signal charge cloud with a position precision of $2.5 \mu \mathrm{m}$ (rms) (see Fig 3). This is achieved by increasing the charge cloud diameter by reducing the electric field during the charge collection process and therefore increasing the charge diffusion process.

All the above improvements are delivering new qualities to the compact X-ray camera stimulating new methods for measurement by (a) saving measurement time, (b) unveiling new properties of the targets under test or (c) developing new measurement methods for basic and applied science or industrial applications.

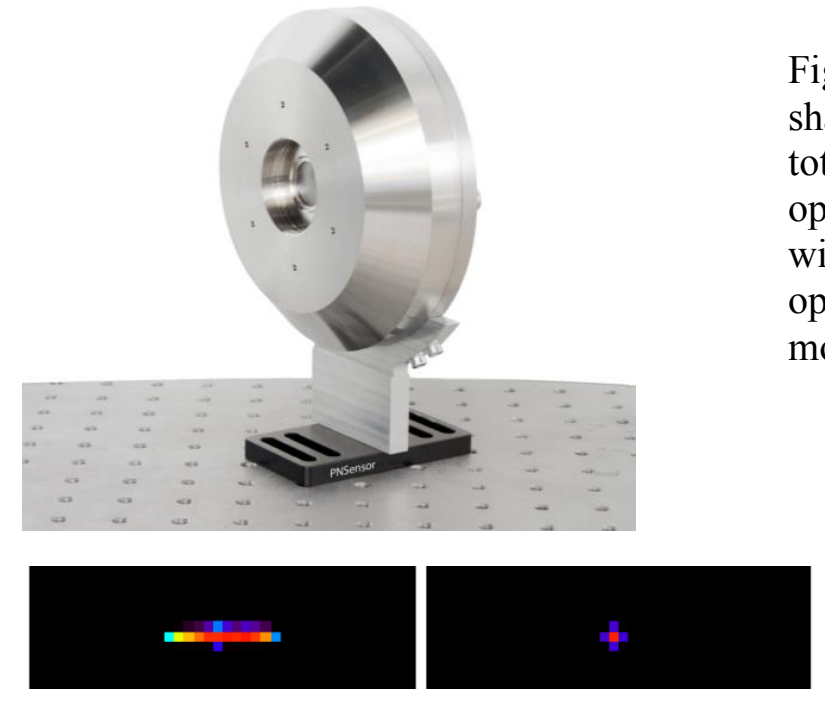

Fig.1: The new Colour X-ray Camera (CXC) has a round shape with a diameter of $14 \mathrm{~cm}$ and a depth of $4 \mathrm{~cm}$. The total weight was substantially reduced to $3 \mathrm{~kg}$. It can be operated in normal environment, e.g. air. In this case a Be window protects the pnCCD inside. A windowless operation in vacuum is possible as well. The CXC has a mount for easy adaptation to other equipment.

Fig. 2: The charge spilling over the pixel boundaries spreads preferentially in the direction perpendicular to the transfer. In the anti-blooming mode all charge is contained in a single pixel.

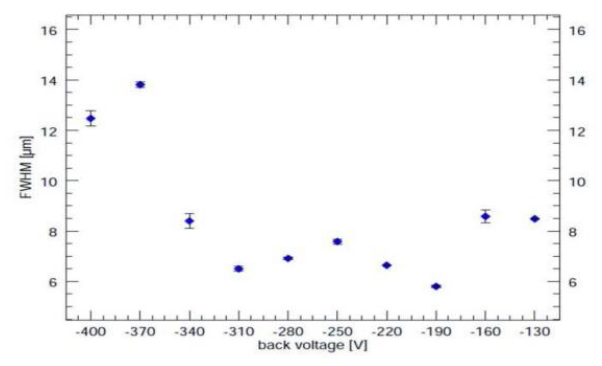

Fig. 3: When radiation hits the detector, a small, very well localized charge cloud is produced through the ionization process. The cloud size increases first through electrostatic repulsion and then through diffusion. When arriving in the potential well of the pixel structure the cloud size may exceed $20 \mu \mathrm{m}$, as a function of the energy of the $\mathrm{X}$-ray and the detector operating conditions. The very low noise enables the user to correctly measure the charge distribution in the pixel structure and find the X-ray interaction point. 